\title{
ESTUDIO DE MERCADO SOBRE LAS NECESIDADES DE PROFESIONALIZACIÓN Y CUALIFICACIÓN DOCENTE EN EL MUNICIPIO DE CÚCUTA
}

Por:

Efrén Alberto González García'

\section{RESUMEN}

Uno de los problemas que no ha resuelto la Facultad de Educación, Artes y Humanidades de la Universidad Francisco de Paula Santander es determinar qué programas de formación de educadores requiere la región, y cuáles son sus necesidades de cualificación y perfeccionamiento que le permita planear estratégicamente su desarrollo e incidir significativamente en el mejoramiento de la calidad de la educación en el Departamento Norte de Santander. Adicionalmente, el relevo generacional exige visionar cuántos Docentes y en qué áreas se deben preparar para los próximos dos quinquenios.

Los programas de pregrado y postgrado que ofrece la Facultad no consultan las verdaderas necesidades de la región y de los Docentes en servicio, lo que explica, de alguna manera, su baja demanda, sin dejar de mencionar, el bajo perfil de la profesión docente y un marco normativo laxo para el ejercicio de la docencia.

La investigación se desarrolló en 44 instituciones del Municipio de Cúcuta, que incluyó a 59 rectores y 700 Docentes.

Los resultados concluyen que la Facultad de Educación, Artes y Humanidades no responde a las necesidades de formación de nuevos Docentes en las áreas fundamentales y que los cursos de actualización docente no atienden las verdaderas debilidades de los educadores en servicio. Las áreas que requieren mayor demanda son las Ciencias Sociales, Filosofía, Idioma Extranjero (Inglés); igual, se requieren administradores escolares para el nivel de pre-escolar, docentes para educación especial y en las áreas de la media Técnica.
Cerca del $60 \%$ de los actuales Docentes se desvincularan, en el próximo decenio, por retiro forzoso o edad de jubilación. No se atiende a la población excepcional ni afroamericana, los discapacitados, la población campesina y desplazados.

Palabras claves: Formación y cualificación docente, demanda de postgrado, estudio de mercado.

\section{ABSTRACT}

One of the problems that have not solved the Faculty of Education, Arts and Humanities is to determine what programs of formation of educators require the region, and which are their necessities of qualification and improvement that allows him to plan strategically its development and to affect the improvement of the quality of the education in the North Department of Santander significantly. Additionally, the generational relief demands to visionary how many Educational and in what areas are due to prepare for next the two quinquenniums.

The programs of pedigree and post degree that the Faculty offers do not consult the true necessities of the region and the Educational ones in service, the one that explains, of some way, its low demand, without letting mention, the low profile of the educational profession and a lax normative frame for the exercise of teaching. The investigation was developed in 44 institutions of the Municipality of Cúcuta that included to 59 directors and 700 Educational ones.

The results conclude that the Faculty of Education, Arts and Humanities does not respond to the new necessities of formation of Educational in the fundamental areas and that the educational advanced training courtesies in good condition do not take care of the true weaknesses of the educators. The areas that require

Profesor adscrito al departamento de pedagogía, Andragogía, comunicación y multimedia. Catedrático del área de Administración y Legislación Educativa en los programas de Licenciatura de la Facultad de Educación, Artes y Humanidades. Doctorando en Calidad y Evaluacion de la Universidad Complutense de Madrid. e-mail: efrenalbertogonzalez@hotmail.com 
Estudio de mercado sobre las necesidades de profesionalización y cualificación docente en el Municipio de Cúcuta

greater demand are Social Sciences, Philosophy, Foreign Language (English); equal, scholastic administrators for the pre-scholastic level, Educational require themselves for special education and in the areas of the average Technique.

Present $60 \%$ of the Educational ones broke contact in the next decade by discharge from military service or age with retirement. It is not taken care of the exceptional nor Afro-American population, the discapacitados ones, and the population farmer, displaced.

\section{INTRODUCCIÓN}

La carencia de un plan estratégico que consulte una visión prospectiva de la Facultad de Educación, Artes y Humanidades ha venido afectando el cumplimiento de su misión de liderar la formación de formadores en la región y el departamento. El estudio de mercado permite revelar las necesidades de formación que requieren los educadores del Municipio de Cúcuta en los próximos años, así como ponderar la vigencia de los actuales programas de formación, son los objetivos centrales de la presente investigación. Se ensambla en un marco teórico que revela la importancia de la capacitación docente en el mejoramiento de la calidad educativa y su influencia en el desarrollo de competencias (no solo para las pruebas SABER, ICFES o ECAES); revisa los enfoques en la fundamentación pedagógica de los educadores y emerge con urgencia la acreditación social, política, económica y cultural de la profesión docente, finalizando con la estructura de un estudio de mercado pertinente. Se presenta un análisis de la Ley 715 del 2001 en lo que respecta a los cambios en los requisitos para el ascenso en el escalafón, su inscripción y evaluación de desempeño.

Uno de los aspectos importantes que toca el estudio se refiere a la calidad. En este sentido, y citando a Toranzos "se reconoce una dimensión políticaideológica del concepto de calidad y una dimensión técnico-pedagógica. La primera se refiere a aquellos aspectos que se vinculan con la definición de los lineamientos generales de la política educativa, y la segunda dimensión a aquellos aspectos vinculados directamente con la operación, el quehacer y la gestión pedagógica directamente"[1]

Resulta interesante incluir elementos sociológicos de la profesión Docente que son determinantes en la escogencia de ella como oficio para toda la vida. Ángel Díaz Barriga afirma que la conjugación de una serie de procesos como la retribución de su trabajo, el reconocimiento social al mismo y los espacios que se le conceden para definir el sentido de su actuación originan los mecanismos de frustración en la tarea Docente [2].

Gimeno Sacristán y Pérez Gómez, identifican en la formación del Profesor una concepción ideológica que denomina "perspectiva tradicional", que refuerza lo expuesto por Weber. Esta concibe la enseñanza como una actividad artesanal y al profesor como un artesano; lo que supone, un oficio no intelectual apartado del rigor científico, que no exige de su ejecutor inversión en investigación, y por tanto, el desarrollo de su tarea es simple y llana [3]. Aunque existen reconocimientos formales a su misión, pareciera que estos reforzarán imágenes de resignación, de héroe nacional o de bajo status social.

Otros como Gloria Ramírez, afirman que la calidad de la educación está directamente articulada con la profesión Docente. Los países con mayor grado de desarrollo educativo son precisamente aquellos que mejor reconocimiento social, cultural y económico le dan a la profesión Docente [4].

\section{MATERIALES Y MÉTODOS}

En lo metodológico se utilizó una estadística descriptiva e inferencial ( $r$ de pearson) para verificar la correlación entre la edad de los Docentes y el tiempo de servicio para efectos de jubilación. Se aplicó una encuesta con tres secciones. La primera se denominó 
Estudio de mercado sobre las necesidades de profesionalización y cualificación docente en el Municipio de Cúcuta

Información General y hace referencia a aspectos como: Institución donde labora, correo electrónico, edad, grado de escalafón, años de servicio oficial. La segunda sección se denominó Forma de Vinculación. $\mathrm{Se}$ clasificaron en: Oficiales (nacionales, nacionalizados, departamentales y municipales); de contrato (departamental, municipal, OPS) y de convenios (Ong's). La tercera sección hace referencia a las necesidades Docentes. Se listaron nueve (9) áreas fundamentales para determinar en cuál de ellas quería vincularse, en el evento de no ser licenciado. Otra parte de la misma sección determina los cursos de cualificación y se listaron 19 opciones, considerando aquellos que la Facultad han venido ofertando. Finalmente, se le presentan las opciones de postgrado para que determine la que potencialmente quisiera estudiar.

El proceso de recolección de información se basó en la organización de las comunas de Cúcuta con la colaboración de los estudiantes de la cátedra de Administración y Legislación Educativa de VI, VII y VIII semestre de las Licenciaturas de Biología y Química y Matemática e Informática del segundo semestre del 2002. Se siguieron los siguientes pasos:

a) Tomar la división por comunas y asignar a los estudiantes dependiendo del lugar en donde residían.

b) Determinar el número de colegios y escuelas fusionadas públicas de cada comuna.

c) Determinar el número de Docentes y directivos de cada colegio y escuela por comuna.

d) Diseño del instrumento con participación de los estudiantes.

e) Capacitación de los mismos para su aplicación.

f) Aplicación de la encuesta.

g) Socialización de los resultados.

h) Elaboración y presentación del informe final.

\section{RESULTADOS}

Se presentan en nueve (9) variables, a saber: escalafón docente, edad, tiempo de servicio prestados al Estado, nivel escolar del docente, Docentes según área de formación, nivel escolar de los rectores, tipo de vinculación laboral, necesidades de formación y cualificación docente y demanda de las especializaciones de la Faculta de Educación, Artes y Humanidades.

Escalafón docente: la totalidad de la muestra esta escalafonada en los términos del Decreto 2277/79, donde el $21 \%$ se ubica en el grado 14 (máxima categoría) la cual se adquiere con experiencia y estudios de postgrado. El $26 \%$ se ubica entre los grados 1 al 9 y el $45 \%$ entre los grados 10 al 13 . (Véase Figura 1)

Figura 1. Escalafón docente

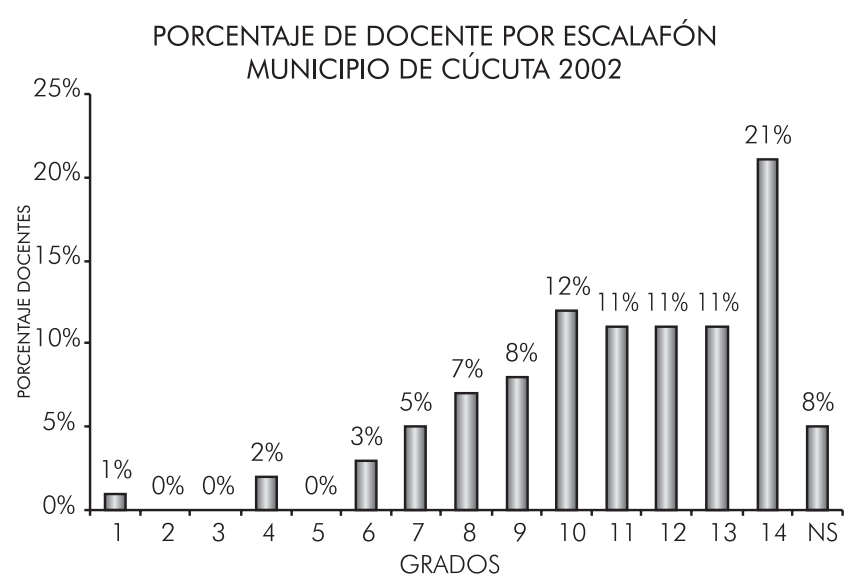

Edad promedio de los educadores oficiales: el $42 \%$ son menores de 40 años, siendo la edad más baja 21 años y la más alta 60 años. El 36\% oscila entre 41 y 50 años y el $18 \%$ entre 51 y 60 años. (Véase Figura 2)

Figura 2. Edad promedio de los educadores oficiales

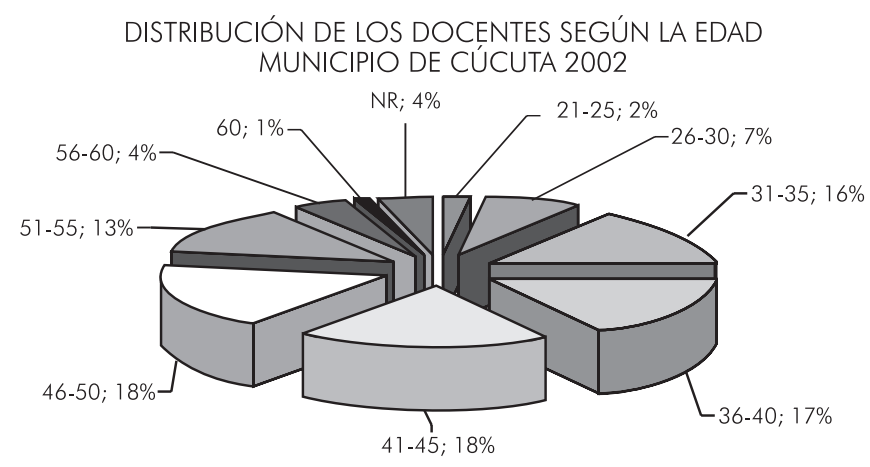


Estudio de mercado sobre las necesidades de profesionalización y cualificación docente en el Municipio de Cúcuta

Años de servicio al estado: El 53\% tiene menos de 20 años de servicio, en tanto que, el 35\% tiene entre 21 y 30 años y el $9.5 \%$ más de 31 años de servicios. Igual, el estudio determinó el total de años de experiencia al magisterio oficial y no oficial encontrando para el primero, que algo más de $32 \%$ tiene entre 1 y 10 años de servicio al Estado, el $21 \%$ entre 11 y 20 años, el 35\% entre 21 y 30 años y $9 \%$ más de 31 años. (ver figura 3)

En relación con los servicios prestados al magisterio no oficial se observa que 19\% tiene entre 1 y 10 años de ser maestro, el 33\% entre 11 y 20 años, el 35\% entre 21 y 30 años y el 12\% más de 35 años.

Figura 3. Distribución de los Docentes según Años de Servicio al Sector Oficial.

DISTRIBUCIÓN DOCENTES SEGÚN AÑOS DE SERVICIO SECTOR OFICIAL MUNICIPIO DE CÚCUTA 2002

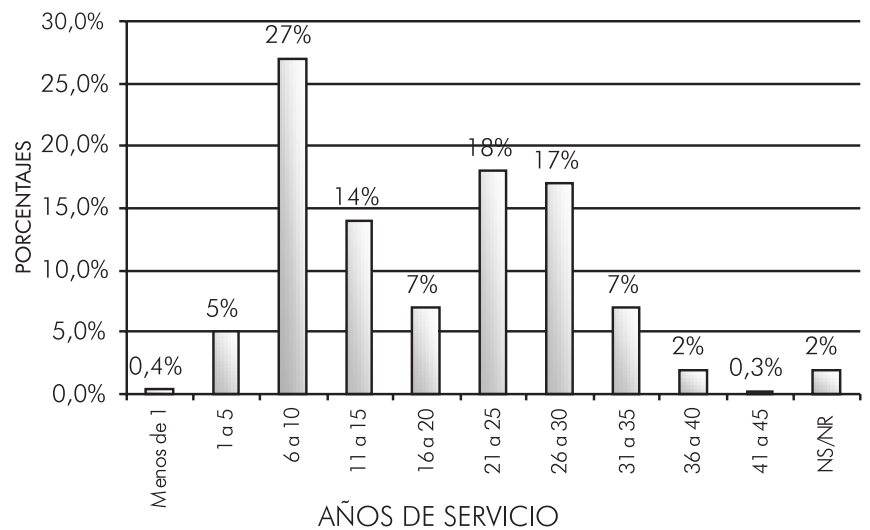

Nivel educativo de los docentes: De 700 Docentes, el $26 \%$ poseen estudios de Licenciatura, el $6 \%$ es bachiller pedagógico, un $8 \%$ no tiene ninguna formación pedagógica y un $11 \%$ no tiene estudios de postgrado en áreas pedagógicas o disciplinarias. (Véase Figura 3)

(Convenciones: $\mathrm{PRI}=$ Primaria, $\mathrm{BR}=$ Bachiller Pedagógico, OTR $=$ Otro tipo de Bachiller, TEC = Tecnólogo en Educación, $\mathrm{OTR}=$ Otro tipo de tecnólogo, PP=Profesional de la Pedagogía (licenciado), OTR = Otro tipo de profesional, POS = Postgrado en Educación, OTR = Otro tipo de Postgrado, N/S= No sabe, no responde.)
Figura 4. Nivel educativo de los Docentes

\section{NIVEL EDUCATIVO DOCENTES MUNICIPIO DE CÚCUTA 2002}

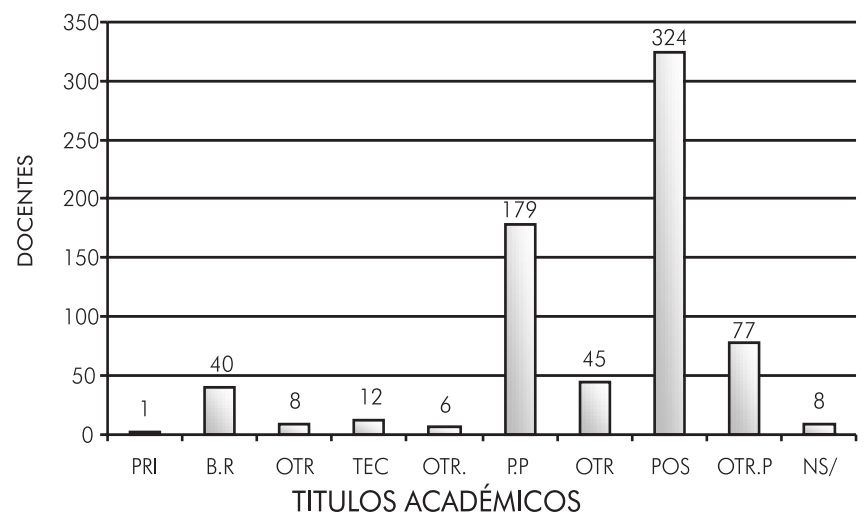

Distribución docente según área de formación: El $68 \%$ de la muestra afirma tener conocimientos sobre un área fundamental, sin embargo, un $69 \%$ no define su área de desempeño. El estudio arroja un déficit de Docentes en áreas como Ciencias Sociales, Educación Artística, Educación Religiosa, Educación Física, Recreación y Deportes, Filosofía e Idioma Extranjero. Debe entenderse que las Instituciones tiene Docentes que atienden estas áreas, pero no tiene una formación epistémica sobre la misma. (Véase Tabla 1.)

Tabla 1. Docentes según Áreas de Formación

\begin{tabular}{|l|c|c|}
\hline \multicolumn{1}{|c|}{ ÁREAS } & NÚMERO DOCENTES & $\%$ \\
\hline C.N. Educación Ambiental & 41 & 9 \\
Ciencias Sociales & 35 & 7 \\
Educación Artística & 42 & 9 \\
Educación Ética y Valores Humanos & 40 & 8 \\
Educación Física, Recreación y Deportes & 27 & 6 \\
Educación Religiosa & 8 & 2 \\
Humanidades, Lengua Cast. Idioma Ext. & 50 & 11 \\
Matemáticas & 39 & 8 \\
Tecnología e Informática & 125 & 26 \\
NS/NR & 69 & 14 \\
\hline
\end{tabular}

Nivel educativo de los rectores: De 59 rectores, el $32 \%$ son licenciados. El 33\% tiene postgrado, el 14\% son normalistas, $7 \%$ son bachilleres pedagógicos y el $5 \%$ tienen título distinto al de licenciado (Véase Figura 4). Se encontró que dentro del grupo de los licenciados éstos se distribuían así: $25 \%$ en Básica Primaria, el $20 \%$ en Biología y Química y el 10\% en Ciencias Sociales (habiendo déficit de maestros en ésta área). 
Estudio de mercado sobre las necesidades de profesionalización y cualificación docente en el Municipio de Cúcuta

Tan solo el $15 \%$ es administrador educativo por formación.

Figura 5. Nivel educativo de los rectores

NIVEL EDUCATIVO DE LOS RECTORES MUNICIPIO DE CÚCUTA 2002

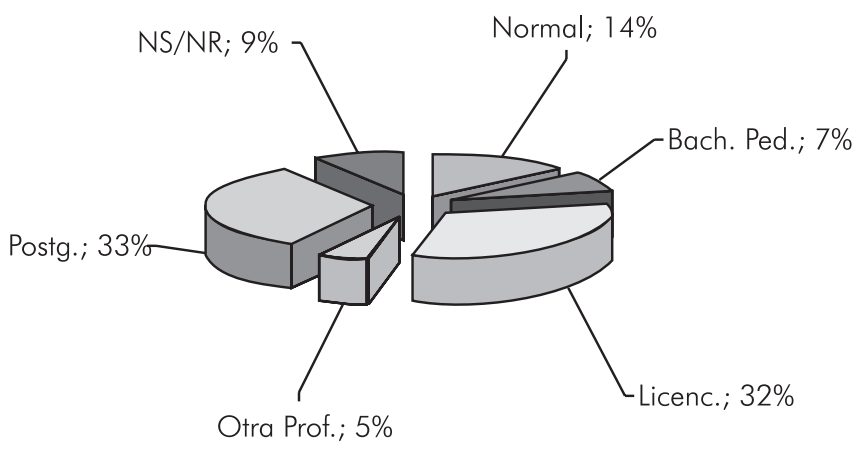

Docentes según el vínculo laboral: El 84\% tiene vinculación oficial, es decir, nombramiento por decreto; el $10 \%$ vinculado por contrato y el $6 \%$ en convenio. La distribución por Comuna arrojó que las 6 y 7 tiene el mayor número de Docentes vinculados por la nación; en tanto que, la Comuna 1 sobresale por tener el mismo número de Docentes contratados, oficiales y de convenio. La Comuna 5 solo tiene Docentes oficiales.

Necesidades de formación y cualificación: De 19 proyectos presentados por la Facultad de Educación los de mayor demanda fueron lo de Evaluación por competencias con un 18\%, psicología del Aprendizaje (9\%), estándares curriculares y Lúdicas con $8 \%$ y los proyectos pedagógicos con $7 \%$.

Demanda de las especializaciones de la Facultad de Educación U.F.P.S.: Se obtuvieron 519 respuestas distribuidas así: el $51 \%$ se inclina por la Informática Educativa, el $19 \%$ por la Orientación Vocacional y el 18\% por Prácticas Pedagógicas Universitarias. El 12\% no respondió.

\section{DISCUSIÓN}

Cuestionable, desde toda óptica, los resultados de la Facultad de Educación de la Universidad
Francisco de Paula Santander, dado su campo disciplinar y la trayectoria en la región. Las razones se pueden encontrar en la ausencia de un proyecto de Facultad que visione a largo plazo las metas y objetivos y organice los recursos para tales propósitos. Esta ausencia de liderazgo institucional en lo educativo es sintomática de los procesos de formación al interior de los programas académicos. La ausencia de colectivos Docentes que indaguen y propongan innovaciones curriculares en los actuales programas aleja a la Facultad de auspiciar investigaciones serias alrededor de la problemática educativa, y es allí, en donde se ha perdido espacio de discusión y debate. El ausentismo en foros regionales y nacionales de la colectividad académica de la facultad raya con las exigencias de mejoramiento que cada vez la ciencia y la tecnología proponen al docente universitario.

El nuevo escenario del marco jurídico de la Ley 715/ 2001 y su Decreto reglamentario 1278 del 2002, no ha sido discutido seriamente al interior de la Facultad y se refiere, precisamente, a la formación de maestros en el nuevo estatuto de profesionalización docente. ¿Qué tipo de maestros se están formando en las aulas? ¿Para qué escenarios? ¿QQué competencias desarrollan los maestros en 10 o 12 semestres? ¿̇Son los actuales postgrados la respuesta a las necesidades Docentes? ¿Consulta los proyectos de actualización los requerimientos de los Docentes? Estas preguntas, entre otras, que serían de obligatorio cuestionamiento en la Facultad, no se realizan, cumpliendo con la formación profesionalizante, más no, con un proyecto educativo académico.

El diseño de un plan de mercadeo que establezca estrategias de oferta de los programas no es una prioridad en la Universidad, ni en la Facultad. El portafolio de servicios no es una herramienta técnica de uso racional para vender servicios y cumplir con los objetivos de extensión; existe la creencia de considerar la naturaleza pública de la Universidad, como único argumento para no esforzarse en innovar, 
Estudio de mercado sobre las necesidades de profesionalización y cualificación docente en el Municipio de Cúcuta

mejorar y ofertar programas de calidad. La amenaza permanente de instituciones nuevas en la región usando tecnología de punta (NTIC) no causa preocupación, en tanto que, los niveles de deserción escolar aumentan en los Programas de Licenciatura. Es urgente que la Facultad de Educación de la U.F.P.S. establezca el "segmento del mercado" al cual le interesan los servicios y precisar, caracterizar al "usuario, cliente o beneficiario típico" de sus programas, so pena de insistir en captar estudiantes para otros programas (usando los propios como trampolines para traslado).

En este momento se deben tener nuevos productos en preparación para reemplazar al que ha alcanzado su ocaso. Debe considerarse que un producto en esta situación puede considerarse todavía lucrativo, y que período de decadencia puede ser de varios años.

Si se mira al interior de la Facultad para explicar las razones de sus resultados, es cuestionable la desatención en la vinculación de talento humano a los departamentos de humanidades y pedagogía, la labor pedagógica recae en once (11) Docentes en propiedad que deben atender, no solo los programas propios de Licenciaturas, sino, los requerimientos de los otros programas en lo que se refiere a la formación humanística, desechada, despreciada y subvalorada por algunos colegas de las llamadas disciplinas duras.

Finalmente, la ausencia de un liderazgo educativo en la región como Facultad de Educación se evidencia en los roles que se desempeñan en los diferentes espacios y agencias del gobierno que deciden sobre lo educativo. El peso político en la administración central de la Universidad es nulo, pero la exigencia de participar en programas y proyectos claves para la administración es perentoria.

\section{CONCLUSIONES}

Los resultados son claros en evidenciar aspectos que, aunque se suponían, expresan una realidad preocupante para el futuro de la Educación Regional: el Municipio de Cúcuta carece de Docentes formados en disciplinas fundamentales que atiendan las necesidades de las nuevas generaciones; algo más, la Facultad de Educación de la Universidad Francisco de Paula Santander, no atiende a las necesidades de los Docentes en servicio y no responde a las necesidades de nuevos maestros en las áreas fundamentales establecidas en la Ley 115 de 1994.

Hingston, establece que un producto o servicio para ofrecerse debe condicionarse a la expansión o contracción de mercado, a su estabilidad o dependencia de otro mercado, que a la vez esté cambiando rápidamente. Los mercados tienden a empezar con lentitud, a expandirse con rapidez y a nivelarse $0, a$ veces, a declinar. Observar el ciclo vital del mercado es clave en el éxito de la oferta y la demanda [5]

Los cursos de actualización no atienden las verdaderas debilidades de los Docentes para mejorar su desempeño en el aula. Se percibe un gran vacío intelectual en la capacitación docente, dado el tipo de cursos o proyectos ofertados por la Facultad de Educación.

Es urgente la formación de Docentes en áreas como las Ciencias Sociales (programa No Acreditado), Educación Religiosa, Educación Artística, Filosofía (se debe diseñar para su acreditación), Idioma Extranjero (se creó un Instituto que no funciona, no tiene presupuesto, ni acciones específicas enfocadas a su funcionamiento; se debe diseñar el programa para su acreditación); Administración Educativa (aunque no es un área fundamental, se requiere formar los directivos de las Instituciones Educativas para los nuevos tiempos); en el nivel de Pre-escolar está todo por hacer. Se requieren docentes para la educación especial, la media técnica y la población campesina, indígena, negra y con problemas de aprendizaje.

En el próximo decenio algo más del $60 \%$ de los Docentes oficiales estarán de retiro forzoso y será 
Estudio de mercado sobre las necesidades de profesionalización y cualificación docente en el Municipio de Cúcuta

necesario tener disponible, la generación de maestros de relevo en todas las áreas.

Los postgrados de la Facultad de Educación no tienen demanda en el contexto regional, lo que exige un estudio más profundo sobre las necesidades al nivel de maestrías y doctorados. Es perentorio que la Facultad pulsee el nuevo marco jurídico de ascenso de los maestros y proponga programas novedosos que apunten a satisfacer el nuevo mercado por venir.

Al respecto, Kotler, define el producto como todo aquello que puede ofrecerse para satisfacer una necesidad o un deseo, los cuales son identificados a partir de una Investigación de Mercado. Por lo tanto, es la identificación de necesidades del consumidor o usuario, la que permite definir el producto o servicio que requiere y comprará el cliente. Un cliente compra solo si tiene una necesidad no cubierta, o un deseo por satisfacer. Aunque esta afirmación parezca obvia, son muchas las Empresas que lo ignoran y siguen empeñadas en vender lo que producen, en vez de producir lo que sus clientes quiere comprar o insisten en trabajar un producto o servicio por el hecho que ha funcionado bien durante años sin tener en cuenta que el mercado ha cambiado. Por tanto, centrar un negocio en un producto o servicio concreto implica que la Empresa está sometida a importantes riesgos tecnológicos y comerciales [6]

No se requiere demasiado análisis para inferir la desarticulación de la Facultad de Educación (sus programas académicos ofertados) con los planes de desarrollo sectorial municipal y departamental; la desatención a los niveles precedentes muestra la ausencia de un proyecto de facultad sintonizada con la región, las instituciones y los Docentes.

La Facultad de Educación de la Universidad Francisco de Paula Santander tiene una herramienta jurídica que le permite direccionar las propuestas de Actualización Docentes y focalizar la población objeto de las mismas, de tal suerte que pueda organizar serias acciones de trabajo colectivo ubicando los docentes y directivos en proyectos institucionales que apunten al mejoramiento cualitativo de Docentes, Directivos y Estudiantes.

Sobre estos cambios, García establece que se acabaron los grandes saltos en el Escalafón Docente, que en otrora, permitían a un Docente pasar de un Categoría Segunda a una Categoría Décima con los costos fiscales conocidos. Igual el cambio en las condiciones para acceder al ascenso por estudios superiores establecido como estímulo en el Artículo 39 del 2277 fue derogado [7]

La Ley 715 establece que en materia de inscripciones y ascensos 'la entidad territorial (certificada) determinará la repartición organizacional..."[9] de conformidad con el reglamento que para tal expida el Gobierno Nacional. Tal repartición organizacional se refiere a las solicitudes de ascenso radicadas antes de la vigencia de la Ley 715 del 2001.

La responsabilidad de organizar el futuro de la formación de nuevos maestros es una labor a tres bandas: Secretarías de Educación, Instituciones y Facultad de Educación, sincronizadas desde la Universidad.

Es vital establecer el "segmento del mercado" al cual le interesan los servicios y precisar, caracterizar al "cliente típico" del negocio. Se debe focalizar un "nicho de mercado" que presente demanda del producto o servicio, que sea suficientemente grande para mantener el negocio y que no esté todavía bajo el dominio de sus competidores. Una concienzuda investigación de mercado es la única forma de establecer con precisión los límites del segmento del mismo.

\section{AGRADECIMIENTOS}

Finalmente, el autor agradece a Revista RESPUESTAS, por su valiosa colaboración y paciencia en la corrección del presente artículo. 
Estudio de mercado sobre las necesidades de profesionalización y cualificación docente en el Municipio de Cúcuta

\section{BIBLIOGRAFÍA}

[1] TORANZOS, Lidia. La calidad como oportunidad de aprendizaje. En: Educación y cultura. $N^{\circ} 60$. Bogotá, 2002.

[2] BARRIGA, Díaz Ángel. La tarea docente. Una perspectiva didáctica grupal y psico-social. México: UNAM, 1993.

[3] GIMENO, Sacristán J., y PÉREZ, Gómez A. I. Comprender y transformar la enseñanza. Madrid: AlfaOmega-Morata, 1999.

[4] RAMÍREZ, Gloria Inés. La educación que queremos. En: Educación y cultura. № 60. Bogotá, 2002

[5] HINGSTON, Peter. Marketing efectivo. México: Prentice Hall, 2002.

[6] MARTíneZ, B. Carmen Elisa. Estrategias de mercado. Bucaramanga: UDES, 2000.

[7] GARCIA, R. Carlos H. Efectos de la Ley 715 en la gestión del sector educativo. Cuadernos educativos № 1. Bogotá: Universidad Autónoma de Colombia. Instituto Superior de Pedagogía, 2003.

[8] FUNDASUPERIOR. Reforma de las competencias y recursos para los sectores sociales en Colombia. Bogotá: Esatc \& Fundasuperior, 2002.

Fecha recibido: Abril 19 de 2005

Fecha aceptación: Junio 22 de 2006 